\title{
The Optimization of Traffic Control Scheme for Emergency Supplies Transportation
}

\author{
Yumei $\mathrm{Li}^{1,4, \mathrm{a}}$, Hongguo Wang ${ }^{2,4^{*}, \mathrm{~b}}$,Ping $\mathrm{Li}^{3,4, \mathrm{c}}$, Zengzhen Shao ${ }^{2,4, \mathrm{~d}}$, and Mingguang \\ $\mathrm{Ma}^{2,4, \mathrm{e}}$ \\ ${ }^{1}$ School of Management Science and Engineering, Shandong Normal University, \\ Jinan, 250014, China \\ ${ }^{2}$ School of Information Science and Engineering, Shandong Normal University, \\ Jinan, 250014, China \\ ${ }^{3}$ School of Mathematical Sciences, Shandong Normal University, Jinan, 250014, \\ China \\ ${ }^{4}$ Shandong Provincial Logistics Optimization and Predictive Engineering \\ Technology Research Center, Jinan, 250014, China \\ assxglym@163.com; ${ }^{b}$ wang666666@126.com; ${ }^{c}$ sdnuliping@163.com;

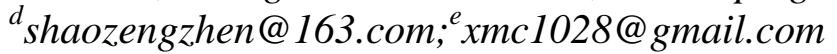

\begin{abstract}
After the disaster, emergency supplies transportation is the key to emergency rescue. In order to ensure the rapid delivery of emergency supplies to the affected area, it is necessary to take appropriate control measures on roads. However, the implementation of traffic control will certainly bring interference for social vehicles. So there is need to optimize the traffic control scheme. In this paper, traffic control for different time intervals is implemented on the road section, and the optimization of traffic control scheme is undertaken. This study includes three parts as following: Firstly, the traffic control model is built to minimize disturbance caused by traffic control under the premise that the time requirement of emergency supplies transportation is met. Secondly, optimization algorithm based on greed is designed to solve the model. Then the concrete traffic control scheme is obtained at last. In practice, the optimization algorithm is proved to be convergent. Finally, the model and algorithm are analyzed and validated by SiouxFall road network. In conclusion, the optimization of traffic control scheme could guarantee the delivery of emergency supplies to the affected area within time limit as well as minimize disturbance caused by traffic control.
\end{abstract}

Keywords: emergency supplies transportation, traffic control scheme, optimization algorithm based on greed

\section{Introduction}

A large quantity of emergency supplies will inevitably be required after the disaster, in order to reduce losses in disasters and speed up the transfer of reconstruction and restoration of production and living order, etc. Since emergency supplies transportation requires a higher time limit, we need to take appropriate control measures on roads during transportation to ensure delivery of emergency supplies to the destination as quickly as possible. However, traffic control will interfere with social vehicles to a certain degree. To ensure the rapid delivery of emergency supplies to the affected areas while minimizing the disturbance, it is necessary to optimize the traffic control scheme.

${ }^{*}$ Corresponding author : Hongguo Wang 
Traffic control as an effective solution to traffic congestion has been widely used. When major events are held, according to the traffic characteristics of the city, implementing corresponding traffic control measures on the road can guarantee the normal operation of traffic during the event $[1,2]$.Traffic control measures on the highway can not only improve the traffic capacity of highway, but also can effectively prevent the occurrence of safety accidents in bad weather conditions [3,4].In urban distribution, traffic control can improve the efficiency of urban distribution, and reduce the interference of freight traffic to urban traffic[5,6].

Yet the traffic control as an impact factor in the studies of emergency rescue has only begun to appear in recent years. A.M. Caunhye, X. Nie, et al. sorted out the optimization model in emergency logistics systematically. They proposed some optimization problems, such as facilities siting, delivery of relief supplies and the wounded, etc. And they also emphasized the significance of traffic control in rescue [7]. By considering the road network partially destroyed by earthquake and by the traffic control executed to avoid traffic congestion, S.L. Li and Z.J. Ma established the multi-objective path selection model for the shortest travel time of relief path and the minimum disturbance caused by traffic control. They designed a two-stage heuristic algorithm to solve that model, which is with great innovation and of practical value [8]. On that basis, they studied the multidepot emergency supplies scheduling optimization problem under traffic control, and designed the no-dominate sort genetics algorithm to solve the model [9]. M.A. Konstantinidou, K.L. Kepaptsoglou, et al. applied traffic control of lane retrograde to emergency relief evacuation, greatly improving the efficiency of rescue [10].

The current studies on emergency rescue based on traffic control mainly focus on specific emergencies, meeting the multi-objective requirement of the shortest rescue time and the minimum disturbance caused by traffic control, giving traffic control schemes, such as the literature [8]. But there is no executable, operational specific schemes for the specific control modes and time of each section.

In order to optimize the scheme of traffic control for emergency supplies transportation, we introduce demi-control mode to control the section for different time intervals. The traffic control model is built to minimize disturbance caused by control within time limit of emergency supplies transportation. Next, by constructing a network graph weighted to time and disturbance, algorithm based on greed is designed to solve the model. Then the concrete traffic control scheme is obtained at last. Finally, the model and algorithm is analyzed and validated by Sioux-Fall road network.

\section{Traffic Control Scheme Optimization Modeling}

\subsection{Problem Statement and Assumptions}

Once the disaster in a region occurs, emergency rescue department will transport emergency supplies to the affected area at the first time. During transportation, due to time constraint of emergency supplies transportation, there is the need for traffic control. However, traffic control will affect the normal operation of social vehicles, disturbing the residents to some extent. So, how to carry out traffic control? Under traffic control, not only the rapid delivery of emergency supplies to the affected area is guaranteed, but also the minimum disturbance due to traffic control. It is necessary to construct model and design algorithm to find the optimal control scheme. Assumptions for the model are defined as follows:

1) Traffic control information is fully disclosed. After the disaster, the emergency management department timely release traffic control information to the public through various channels, and the traveler can adjust their plan according to the information; 
2) According to Wardrop equilibrium guideline, it is assumed that travelers always select the shortest as their path [11];

3) Suppose only a certain type of relief supplies is delivered;

4) Transportation demand is known, obtained by conventional traffic statistics;

5) There are three control modes for sections, namely full control, demi-control, and no control. Under full control, only emergency vehicles are allowed; under demi-control, emergency vehicles in the same direction get through half of the lanes, while social vehicles the left; without control, all the vehicles pass through the section.

\subsection{Problem Formalization}

For the convenience of modeling, this paper will abstract road network as graph $G(V, E)$. Where, $V$ is the set of node $i$ in the network, $i \in\{1,2, \ldots, n\}$, and $E$ represents set of section connecting nodes, $(i, j) \in E$. In addition, the path consists of several sections. The important variables are defined as follows:

$T:$ the arrival time of emergency supplies required;

$T^{\prime}:$ the travel time of rescue vehicles;

$O$ : the supply point set of emergency supplies, $O \subseteq V$;

$D$ : the demand point set of emergency supplies, $D \subseteq V$;

$r$ : a supply start node of emergency supplies, $r \in O$;

$s:$ a demand point of emergency supplies, $s \in D$;

$\mathcal{Q}_{s}:$ the traffic demand of $O D$ between $r$ and $s, r \in O, s \in D$;

$\omega_{(i, j)}^{\varphi}$ : the type of traffic control for section $(i, j)$. When $\varphi=1$, it represents full control for the section, signing $\omega_{(i, j)}^{1}=1$; when $\varphi=2$, it denotes demi-control for the section, signing $\omega_{(i, j)}^{2}=\frac{1}{2}$; when $\varphi=3$, it implies no control for the section, $\operatorname{signing} \omega_{(i, j)}^{3}=0$;

$x_{(i, j)}^{\prime}:$ the traffic flow of section $(i, j)$ under no control, $(i, j) \in E$;

$x_{(i, j)}$ : the traffic flow of section $(i, j)$ under control, $(i, j) \in E$. Under full control, there is no social vehicles in the section; under demi-control, given the probability of bypassing for social vehicles, it is assumed that the flow of social vehicles is $80 \%$ of that under no control. Meanwhile, the road capacity is reduced by half;

$t_{(i, j)}(x)$ : the travel time function of section $(i, j) ;$

$R_{r s}:$ the vehicle path set of $O D$ between $r$ and $s$;

$f_{R r s}^{k}$ : the flow of the $k$-th path in the travel path set $R_{r s}, k \in R_{r s}$;

$\sigma_{R_{r s}}^{(i, j), k}:$ the correlation coefficient between path and section. If section $(i, j)$ belongs to the $k$-th path in the travel path set $R_{r s}$, it is 1 , otherwise, 0 .

\subsection{Objective Function}


In order to achieve the goal of minimizing the disturbance for the social vehicles within time limit of emergency supplies transportation, we define that for the social vehicles the disturbance is the ratio of delay time caused by control to travel time without control. Details are as follows:

1) Without control, all the vehicles pass through the section. For the social vehicles, there is no delay, and the impact is so little that it can be negligible, denoted as 0 ;

2) Under demi-control, the delay time is the difference between travel time under demi-control and that without control;

3) With full control, the delay time is the time that emergency vehicles get through the section;

4) Disturbance caused by traffic control with different time intervals for the path is the sum of disturbance for the section. Expressions detailed are as follows:

$$
Z=\sum_{(i, j) \in R_{r s}} \frac{\left(2 \omega_{(i, j)}^{2} \int_{0}^{x_{(i, j)}} t_{(i, j)}(x) d x-\left(1-\omega_{(i, j)}^{3}\right) \int_{0}^{x_{(i, j)}} t_{(i, j)}(x) d x\right)+t_{(i, j)}(0)}{\left(1-\omega_{(i, j)}^{3}\right) \int_{0}^{x_{(i, j)}} t_{(i, j)}(x) d x}
$$

Simplification is:

$$
Z=\sum_{(i, j) \in R_{r s}} \frac{\left(\int_{0}^{x_{(i, j)}} t_{(i, j)}(x) d x-\int_{0}^{x_{(i, j)}^{\prime}} t_{(i, j)}(x) d x\right)+t_{(i, j)}(0)}{\int_{0}^{x_{(i, j)}} t_{(i, j)}(x) d x}
$$

So the objective function is:

$$
Z_{1}=\min \left\{\sum_{(i, j) \in R_{r s}} \frac{\left(\int_{0}^{x_{(i, j)}} t_{(i, j)}(x) d x-\int_{0}^{x_{(i, j)}} t_{(i, j)}(x) d x\right)+t_{(i, j)}(0)}{\int_{0}^{x^{\prime}(i, j)} t_{(i, j)}(x) d x}\right\}
$$

Formula (1) means the minimum disturbance caused by traffic control for social vehicles under the time requirement.

\subsection{Constraint Condition}

Constraint conditions are as below:

$$
\begin{aligned}
& T^{\prime}=\sum_{(i, j) \in R_{r s}}\left(2 t_{(i, j)}(0)+\int_{0}^{x_{(i, j)}} t_{(i, j)}(x) d x\right) \leq T \\
& \sum_{k \dot{R} R r s} f_{R_{r s}}^{k}=Q_{r s} \quad \forall r s \\
& x_{(i, j)}=\sum_{r \grave{O} O} \sum_{s i \dot{D} D} \sum_{k \dot{o} R_{r s}} f_{R_{r s}}^{k} \delta_{R_{r s}}^{(i, j), k} \quad \forall r s, \forall i, j \in V, \forall(i, j) \in E
\end{aligned}
$$

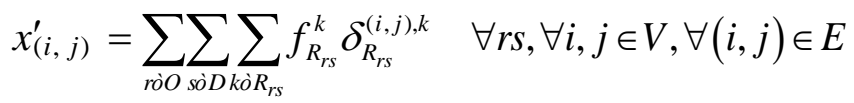

$$
\begin{aligned}
& \sum_{j:(i, j) \in E} \delta_{R_{r s}}^{(i, j), k}-\sum_{j:(i, j) \in E} \delta_{R_{r s}}^{(j, i), k}=\left\{\begin{array}{c}
1, i=r \\
0, i \in V /\{r, s\} \\
-1, i=s
\end{array}\right\} \quad \forall i, j \in V \\
& \sum_{j:(i, j) \in E} f_{R_{r s}}^{k} \delta_{R_{r s}}^{(i, j), k}-\sum_{j:(i, j) \in E} f_{R_{r s}}^{k} \delta_{R_{r s}}^{(j, i), k}=\left\{\begin{array}{c}
f_{R_{r s}}^{k}, i=r \\
0, i \in V /\{r, s\} \\
-f_{R_{r s}}^{k}, i=s
\end{array}\right\} \forall k, \forall r s, \forall i, j \in V \\
& \sum_{r \in O} \sum_{s \in D \in R_{r s}} \sum_{j:(i, j) \in E} f_{R_{r s}}^{k} \delta_{r_{r s}}^{(i, j), k}-\sum_{r \in O} \sum_{s \in D} \sum_{k \in R_{r s}} \sum_{j:(i, j) \in E} f_{R_{r s}}^{k} \delta_{R_{r s}}^{(j, i), k}=0 \quad \forall i, j \in N /\{r, s\}
\end{aligned}
$$




$$
\begin{gathered}
f_{R_{r s}}^{k} \geq 0 \quad \forall r s \\
\delta_{R_{r s}}^{(i, j), k}=\{0,1\} \\
\omega_{(i, j)}^{\varphi}=\left\{\begin{array}{l}
1, \varphi=1 \\
\frac{1}{2}, \varphi=2 \\
0, \varphi=3
\end{array}\right.
\end{gathered}
$$

Formula (2) suggests that travel time of emergency vehicles under control meets the requirement of emergency supplies transportation. Generally speaking, there are two cases for travel time of emergency vehicles. One is the free flow time by control, where control includes full control and demi-control. The other one is obtained by flow without control. The formula is simplified by $\left(2^{\prime}\right)$, namely,

$$
\left.T^{\prime}=\sum_{(i, j) \in R_{r s}}\left(\omega_{(i, j)}^{1}+\frac{1}{\omega_{(i, j)}^{2}} \omega_{(i, j)}^{2}\right) t_{(i, j)}(0)+\left(1-\omega_{(i, j)}^{3}\right) \int_{0}^{x_{(i, j)}} t_{(i, j)}(x) d x\right) \leq T
$$

Formula (3) means demand balance between OD. Formula (4) and (5) are the section flow formula before and after control. Formula (6) indicates user path continuity constraints. Formula (7) denotes user path flow conservation constraints. Formula (8) means flow conservation of section. Formula (9) is non-negative constraint. Formula (10) is 0-1constraint. Formula (11) is the coefficient corresponding to control modes.

\subsection{Travel Time Function}

During traffic flow assignment, there is correlation between vehicle travel time and flow, usually denoted by impedance function $t_{(i, j)}(x)$. The function is mainly used to describe the performance of vehicle operation that may occur in a variety of traffic and road conditions. It is originated from the relationship between per unit time and average speed in the road network. The function is performed by capacity restrictions when flow is assigned to each section. Here, we use BPR function from Federal Highway Administration [12], and the expression is:

$$
t_{(i, j)}\left(x_{(i, j)}\right)=t_{(i, j)}(0)\left[1+\alpha\left(\frac{x_{(i, j)}}{C_{(i, j)}}\right)^{\beta}\right]
$$

Where, $\alpha, \beta$ is regression coefficient, and the typical value is $\alpha=0.15, \beta=4$. $t_{(i, j)}(0)$ is free-flow travel time without resistance for section $(i, j) . C_{(i, j)}$ is the capacity of section $(i, j)$.

For traffic control studied here, according to equation (3) and (4), there is some capacity constraints for the road network. During traffic assignment, only the change of travel time is considered using the Frank-Wolfe algorithm [13] while serious congestion caused by flow beyond section capacity is ignored, which is not allowed in emergency rescue [8]. It is assumed that travel time of control section is free flow time. $x_{(i, j)}$ is the allocated capacity of the section. When $x_{(i, j)} \geq C_{(i, j)}$, travel time of section tends to infinity, namely, $t_{(i, j)}\left(x_{(i, j)}\right)=t_{(i, j)}\left(C_{(i, j)}\right) \rightarrow \infty$, which indicates the section is closed. When $x_{(i, j)}<C_{(i, j)}$, travel time function is expressed as below.

For the emergency vehicles, 


$$
t_{(i, j)}\left(x_{(i, j)}\right)=\left\{\begin{array}{c}
t_{(i, j)}(0), \omega_{(i, j)}^{1}=1 \\
t_{(i, j)}(0), \omega_{(i, j)}^{2}=\frac{1}{2} \\
t_{(i, j)}(0)\left[1+\alpha\left(\frac{\left.\left.x_{(i, j)}\right)_{(i, j)}^{\beta}\right], \omega_{(i, j)}^{3}=0}{C_{i}}=0\right.\right.
\end{array}\right.
$$

For the social vehicles,

$$
t_{(i, j)}\left(x_{(i, j)}\right)=\left\{\begin{array}{c}
t_{(i, j)}(0)\left[1+\left[1+\alpha\left(\frac{x_{(i, j)}}{C_{(i, j)}}\right)^{\beta}\right]\right], \omega_{(i, j)}^{1}=1 \\
t_{(i, j)}(0)\left[\begin{array}{c}
0.8 x_{(i, j)} \\
1+\alpha\left(\frac{1}{-} C_{(i, j)}\right.
\end{array}\right], \omega_{(i, j)}^{2}=\frac{1}{2} \\
t_{(i, j)}(0)\left[1+\alpha\left(\frac{x_{(i, j)}}{C_{(i, j)}}\right)^{\beta}\right], \omega_{(i, j)}^{3}=0
\end{array}\right.
$$

\section{Algorithm for Model}

$$
\left\{\begin{array}{c}
\min Z(x)=\sum_{a} \int_{0}^{x_{a}} t_{a}(x) d x \\
\text { s.t. } \quad \sum_{k} f_{k}^{r s}=q_{r s}, \forall r, s \\
x_{a}=\sum_{r} \sum_{s} \sum_{k} f_{k}^{r s} \cdot \sigma_{a, k}^{r s}, \forall a \\
f_{k}^{r s} \geq 0 \quad \forall k, r, s
\end{array}\right.
$$

Where, $x_{a}$ is traffic flow of section $a ; t_{a}$ is expense of section $a$ (usually measured by time); $f_{k}^{r s}$ is the flow of a path $k$ from supply point to disaster point; $q_{r s}$ means all traffic demand from $r$ to $s$ during the time period studied, namely the amount of $O D$.

This model has proven to be convex programming model [14], so it has a global optimal solution. Nonnegativity and monotonically increasing of section expense function is the sufficient condition for model (3-1) with a unique solution. It is evident that BPR function clearly is with such properties in the practical application. However, this convexity is not suitable for path flow [15].The non-uniqueness of the optimal solution of path flow will eventually become a serious obstacle in descent algorithm application when we solve the problem of traffic network design [16].

To solve this model, many scholars have designed algorithms, such as Frank-Wolfe, Logit equilibrium and variational inequalities, etc. In this paper, combining the objective function with section characteristics under the target, on the basis of Frank-Wolfe, algorithm is designed to solve the model.

\subsection{Optimization Algorithm Based on Greed}

Details are as follows.

Step1: Find the shortest path between $O D$. In the network $G$, according to the free flow time $t_{(i, j)}(0)$ of each section, find the shortest path $P_{1}$ using Dijkstra algorithm;

Step2: Construct network $G^{\prime}$. Carry out full control, demi-control, no control for each section in the path $P_{1} . P_{1}$ as the unregulated edge, add parallel full control and demicontrol edges to configure network $G^{\prime}$ (Figure 1); 
Step3: Calculate the disturbance $z_{i j}$ and travel time $t_{i j}$ of each newly-added edge in the network $G^{\prime}$ (the edge $G^{\prime}-G$ ). Arrange the edges in non-descending order in terms of disturbance $z_{i j}$.If disturbance of section are the same, arrange the edges in non-descending order according to disturbance of per unit time $z_{i j}$, getting sequence $P$;

Step4: $\operatorname{sign} G^{\prime \prime}=G$;

Step5: Add the edge e one by one from P for $G^{\prime \prime}, G^{\prime \prime}=G+\{e\}$.First, we use Dijkstra algorithm weighted to travel time of emergency vehicle to find the shortest path between $O D$. Then, calculate travel time of emergency vehicles $T^{\prime}$ and disturbance for social vehicles Z;

Step6: If the travel time of emergency vehicles $T^{\prime}$ is not greater than the latest arrival time $T$, which is $T-T^{\prime} \leq \varepsilon$, stop adding the edge, and output the control scheme and the shortest path of emergency vehicles. Otherwise, turn to Step5.

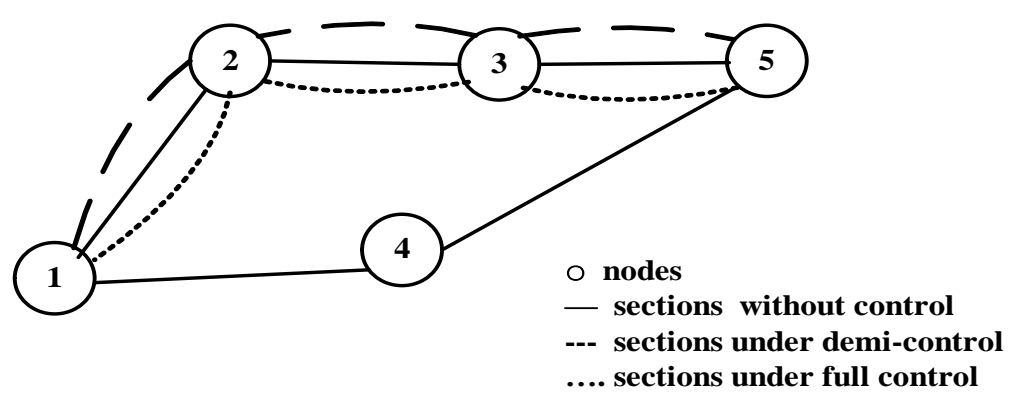

Figure 1. Network $G^{\prime}$

\subsection{Calculation of Travel Time and Disturbance}

Calculate travel time of sections, disturbance and disturbance of per unit time under different circumstances. For emergency and social vehicles, details are as follows under control over sections.

1) Without control, mixed vehicles pass the section. Using the Frank-Wolfe algorithm [13], we can get the result that traffic flow of each section is $x_{(i, j)}^{\prime}$, travel time of section is $\int_{0}^{x_{(i, j)}^{\prime}} t_{(i, j)}(x) d x$, disturbance is 0 , and disturbance of per unit time is 0 ;

2) Under demi-control, rescue vehicles pass through a dedicated lane. Travel time of emergency vehicles is the free flow time $t_{(i, j)}(0)$. Travel time of social vehicles is $\int_{0}^{x_{(i, j)}} t_{(i, j)}(x) d x$. Disturbance is $\mathrm{z}_{i j}=\frac{\int_{0}^{x_{(i, j)}} t_{(i, j)}(x) d x-\int_{0}^{x_{i, j}^{\prime}} t_{(i, j)}(x) d x}{\int_{0}^{\left.x_{i, j}^{\prime}\right)} t_{(i, j)}(x) d x}$. Disturbance of per unit time is $z_{i j}^{\prime}=\frac{\mathbf{z}_{i j}}{t_{(i, j)}(0)}$; 
3)With full control, travel time of emergency vehicles is $t_{(i, j)}(0)$.Social vehicles couldn't get through the section. Disturbance is $z_{i j}=\frac{t_{(i, j)}(0)}{\int_{0}^{x_{(i, j)}} t_{(i, j)}(x) d x}$. Disturbance of per unit time is $z_{i j}^{\prime}=\frac{1}{\int_{0}^{x_{(i, j)}^{\prime}} t_{(i, j)}(x) d x}$.

\subsection{Proof of Convergence}

1) Stay ahead. At each substep in Step5 of the algorithm, only the section with minimum disturbance can be chosen to control. That is, $\omega(i) \leq o(i)$, where $\omega$ represents the current strategy, and $o$ is the optimal strategy. Since the process of adding edges adopts the thought of greedy algorithm, the basic assumption is that at least the current policy and the optimal strategy will ensure the same effect [17].

2) Prove that this condition is satisfied in every substep of Step5. Using deductive reasoning, prove the basic case. Because the first section to be controlled is from the one with the minimum disturbance. It is clearly established. Next, prove that the former $K$ road sections are satisfied, so is the $(K+1)$ one. There are two cases. When section $(K+$ 1 ) is under control, travel time of emergency vehicle is smaller than the latest arrival time of emergency supplies, then the section meets the requirement. Otherwise, when section $(K+1)$ is under control, travel time of emergency vehicle is greater than the latest arrival time of emergency supplies, then it does not meet the requirement. Therefore, the next section chosen to be controlled is the one with minimum disturbance still.

3) Use absurdity to prove that control program with the minimum disturbance can be obtained from our strategy. Because every step only the edge with minimum disturbance can be added. It is assumed that the former $K$ section is established. When edge $(K+1)$ is added, the control program meets the time requirement, but disturbance is not the minimum. Therefore, in the optimum program there must be an edge not in the control schemes obtained, and its disturbance is less than the former $(K+1)$ edges, which conflicts with the bordered strategy.

4) Thus, the proof is in the end. The optimal solution can be obtained from the algorithm.

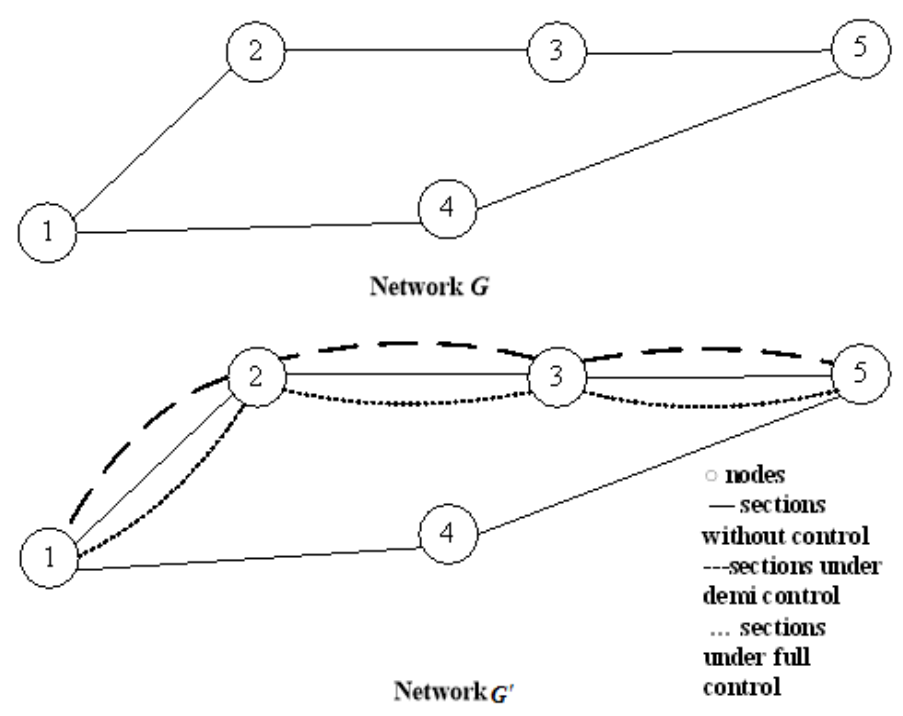




\subsection{Algorithm Complexity Analysis}

In network $G$, it is assumed that travel time of all sections without control is known. According to the description in 3.1, the main step and complexity analysis of the algorithm are as follows:

Using Dijkstra algorithm to find the shortest path $P_{1}$ between $O D$, the complexity of the step is $O\left(n^{2}\right)$. Adding control edges to the section, construct network graph $G^{\prime}$, the complexity of which is $O(n)$. Disturbance and travel time of the newly-added edges is calculated. And sequence $P$ is obtained by the arrangement of the edges in order of disturbance. Its complexity is $O(n \log n)$. According to the sequence $P$, the edge to network $G$ is added one by one, disturbance and travel time is calculated, and then the control scheme is obtained. The complexity is $O\left(n^{3}\right)$. All in all, the overall complexity of the algorithm is $O\left(n^{2}+n+n \log n+n^{3}\right)$, no more than $O\left(n^{3}\right)$.

\section{Numerical Experiment}

In this paper, the Sioux-Fall Road Network topology abstracted from the US Northridge earthquake situation is employed to verify the algorithm [18]. There are 24 nodes, 76 sections, and the topology is shown in Figure 2. Capacity of each link (in $5000 \mathrm{Vol} / \mathrm{h}$, per hour 5000 standard car) and travel time (h) is marked in Figure 2, the format "link number (link capacity, travel time)." Traffic demand between each OD pair is shown in Table 1 .

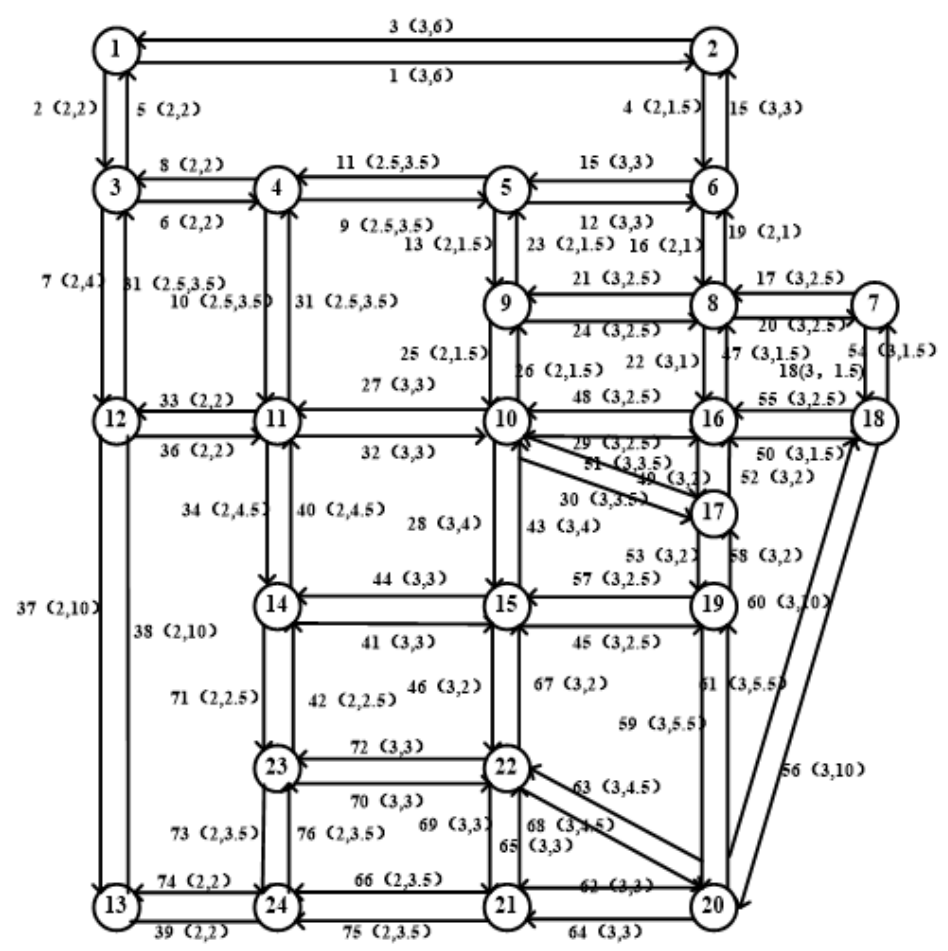

Figure 2. Sioux-Fall Road Network Topology 
Table 1. Traffic Demand of Each OD Pair (unit: 100vol/h)

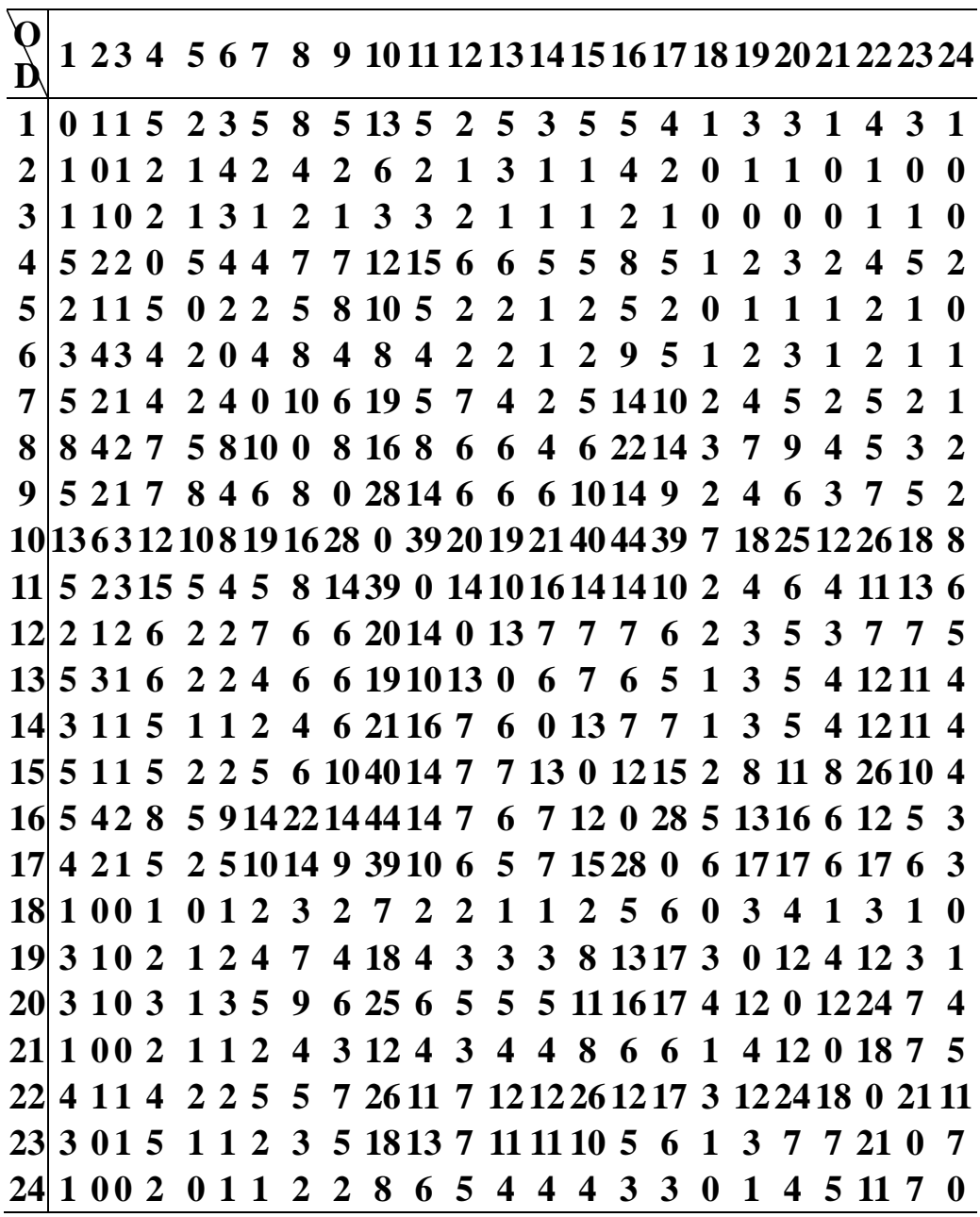

According to the data above, under free flow circumstances, the shortest relief path from node 1 to 20 is 1-2-6-8-16-17-19-20, costing 19 hours. Without control, travel time of emergency vehicles is the longest while disturbance is the minimal. In the case of control, travel time of emergency vehicles is the shortest, that is, free flow of time. However, there is disturbance to some degree. Accordingly, we can control the section for different time intervals, and optimize the traffic control scheme. Different traffic control strategies will be obtained on terms of the latest arrival time requirement of emergency vehicles with supplies. Algorithm experiment is programmed on the platform of Python2.7.10 and PyCharm5.0 platform, running on the machine of the Intel (R) Celeron (R) CPU G540 2.50GHz, 4G memory. Experiment results are shown in the table below.

Obtained from the experiment, the path $P_{1}$ is 1-2-6-8-16-17-19-20.The disturbance sequence for sections added with full control and demi-control edges are shown in Table 2. Table 3 gives the traffic control strategy corresponding to the one with the minimum disturbance under different arrival time constraint. Here, disturbance caused by traffic control strategy is normalized. It is the ratio of disturbance caused by a traffic control strategy to the maximum disturbance of all the strategies. 
Table 2. Sections Sorted by Disturbance

\begin{tabular}{|c|c|c|c|c|c|}
\hline $\begin{array}{l}\text { Disturbance } \\
\text { sequence }\end{array}$ & Section & $\begin{array}{l}\text { Control } \\
\text { mode }\end{array}$ & $\begin{array}{l}\text { Travel time } \\
\text { without } \\
\text { control (h) }\end{array}$ & $\begin{array}{l}\text { Free flow } \\
\text { time(h) }\end{array}$ & $\begin{array}{l}\text { Disturbance under } \\
\text { control for different } \\
\text { time intervals }(\%)\end{array}$ \\
\hline 1 & $1-2$ & $\begin{array}{l}\text { Demi - } \\
\text { control }\end{array}$ & 6.004 & 6 & 0.34 \\
\hline 2 & $2-6$ & $\begin{array}{l}\text { Demi- } \\
\text { control }\end{array}$ & 1.543 & 1.5 & 15.37 \\
\hline 3 & $16-17$ & $\begin{array}{l}\text { Full } \\
\text { control }\end{array}$ & 5.965 & 2 & 33.53 \\
\hline 4 & $8-16$ & $\begin{array}{l}\text { Demi- } \\
\text { control }\end{array}$ & 1.087 & 1 & 44.57 \\
\hline 5 & $17-19$ & $\begin{array}{l}\text { Full } \\
\text { control }\end{array}$ & 3.874 & 2 & 51.62 \\
\hline 6 & $19-20$ & $\begin{array}{l}\text { Full } \\
\text { control }\end{array}$ & 10.140 & 5.5 & 54.24 \\
\hline 7 & $6-8$ & $\begin{array}{c}\text { Full } \\
\text { control }\end{array}$ & 1.759 & 1 & 56.84 \\
\hline
\end{tabular}

For Table 2, take the first row for example. For all newly-added edges, disturbance is the minimum when section 1-2 is under demi-control. Travel time of emergency vehicles through the section is the free flow time $6 \mathrm{~h}$. Disturbance for social vehicles caused by demi-control is $0.34 \%$. Without control, travel time of vehicles is $6.004 \mathrm{~h}$.

Table 3. Traffic Control Strategy with the Minimum Disturbance under different Arrival Time Requirement

\begin{tabular}{ccccc}
\hline $\begin{array}{c}\text { Arrival time } \\
\text { requirement }(\mathbf{h})\end{array}$ & $\begin{array}{c}\text { Transportation } \\
\text { time(h) }\end{array}$ & $\begin{array}{c}\text { Disturbance } \\
(\mathbf{\%})\end{array}$ & $\begin{array}{c}\text { Traffic control strategy (from } \\
\text { node 1 to 20) } \\
\text { Demi control }\end{array}$ & Full control \\
\hline $\mathbf{2 6 . 0} \sim \mathbf{3 1 . 0}$ & 30.37 & 0.13 & $1-2$ & \\
& 30.33 & 6.12 & $1-2,2-6$ & \\
& 26.36 & 19.20 & $1-2,2-6$ & $16-17$ \\
& 26.27 & 36.57 & $1-2,2-6,8-16$ & $16-17$ \\
& 24.40 & 56.69 & $1-2,2-6,8-16$ & $16-17,17-19$ \\
& 19.76 & 77.84 & $1-2,2-6,8-16$ & $16-17,17-19,19-20$ \\
& 19.00 & 100 & $1-2,2-6,8-16$ & $6-8,16-17,17-19,19-$ \\
\hline
\end{tabular}

For Table 3, take the third row for example. When the latest arrival time range of emergency vehicles is $(26.0 \sim 31.0) \mathrm{h}$, according to section sequence, section $1-2$ is in demi-control, while the remaining sections in non-regulated status. Once emergency vehicles pass through section 1-2, remove the control. And then section 2-6 is in demicontrol, while the remaining sections in non-regulated status. As soon as emergency vehicles get through section 2-6, lift the control. When emergency vehicles is about to reach node 16, take section 16-17 in full control, while the remaining sections are in nonregulated status. When emergency vehicles pass through section $16-17$, remove the control. The Travel time from node 1 to 20 is $26.36 \mathrm{~h}$, and disturbance generated is $19.20 \%$. The whole process of traffic control for different time intervals from node 1 to 20 is shown as below. 


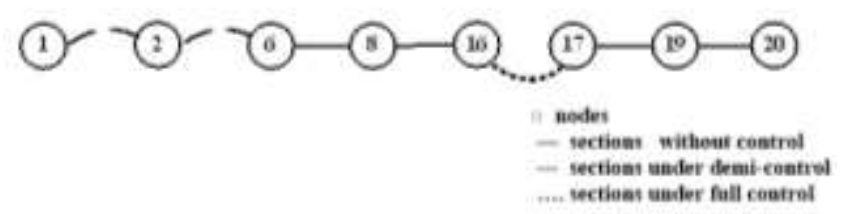

Figure 3. Concrete Control Operation Scheme

In conclusion, with the growth of the latest arrival time of emergency vehicles, fewer sections are in need of traffic control, and disturbance caused by control gradually decreases. When the latest arrival time requirement is not critical, we can take only one section in demi-control, and disturbance of this time can almost be negligible. It can be seen that traffic control is an effective measure to ensure emergency supplies transportation. The demi-control mode can improve the efficiency of traffic control. However, disturbance caused by control occurs. Experimental results show that the optimization of traffic control scheme can not only ensure the emergency supplies transportation but also minimize the disturbance.

\section{Conclusions}

In the background of emergency supplies transportation, we optimize the traffic control scheme so as to ensure the rapid delivery of emergency supplies as well as minimize disturbance caused by traffic control. Considering the specific traffic control mode of each section, the demi-control mode is introduced to control the section for different time intervals. We built the traffic control model to minimize disturbance caused by traffic control on condition that the time requirement of delivery of emergency supplies to the affected area is met. Then, algorithm based on greed is designed to solve the model and obtain the traffic control scheme. Meanwhile, the algorithm is proved to be convergent. That is to say, the optimal strategy could be obtained by the algorithm. At last, the SiouxFall road network is employed to verify the effectiveness of the algorithm. Results show that the optimization of traffic control scheme could guarantee emergency supplies transportation as well as minimize disturbance caused by traffic control, which provides effective theoretical support for emergency rescue work.

\section{Acknowledgments}

This work was supported by China Postdoctoral Science Foundation Project(No.2016M592697), Science and technology development project of Shandong Province(No.2014GGH201022), Soft science project of Shandong Economic and Information Technology(No.2015EI010).

\section{References}

[1] B. H. Mao, J. Y. Guo, Z. Q. Chen and L. P. Gao, "Research on Traffic Schemes for Special Important Activities", Journal of Transportation Systems Engineering and Information Technology, vol. 7, no. 1, (2007), pp.18-24.

[2] L. H. Geng, "Study on Traffic Demand and Evacuation in Urban Large-Scale Event", Master degree thesis, Wuhan University of Technology, (2009).

[3] X. Y. Lu, P. Varaiya, R. Horowitz, D. Su and S. Shaldover, "Novel Freeway Traffic Control with Variable Speed Limit and Coordinated Ramp Metering", Transportation Research Record: Journal of the Transportation Research Board, vol. 2229, no.1,(2011), pp.55-65.

[4] G. Y. Duan, X. L. Zhu and Z. Y. Shen, "Discussions on Traffic Control Ways and Disposal Measures for Expressways in Fog", Technology of Highway and Transport, no. 2, (2004).

[5] Y. C. Hu, "Studies on Sustainable Development of City Logistics-Studies on Development of City Distribution under Urban Freight Traffic Restriction Policies", Doctoral dissertation, Beijing Jiaotong University,(2013). 
[6] X. F. Ji, Y. M. Pu, F. W. Liang and W. W Qin, "Bi-level Programming Model for City Logistics Distribution Optimization under Time Restrict Condition Caused by Traffic Control", Journal of Highway and Transportation Research and Development, vol. 31, no. 12, (2014), pp. 145-152.

[7] A. M. Caunhye, X .Nie and S. Pokharel, "Optimization Models in Emergency Logistics: A literature review”, Socio-Economic Planning Sciences, vol. 46, no. 1, (2012), pp.4-13.

[8] S. L. Li and Z. J. Ma, "User Equilibrium-based Post-earthquake Relief Routing Problems under Traffic Control", Journal of Industrial Engineering and Engineering Management, vol. 28, no. 3, (2014), pp. 148-155.

[9] S. L. Li and Z. J. Ma, "Post-earthquake Multi-depot Relief Distribution under Traffic Control", Journal of management sciences in China, vol. 17, no. 5, (2014), pp. 1-13.

[10] M. A. Konstantinidou, K. L .Kepaptsoglou, M. G .Karlaftis and A. Stathopoulos, "Joint Evacuation and Emergency Traffic Management Model with Consideration of Emergency Response Needs", Transportation Research Record: Journal of the Transportation Research Board, no. 2532, (2015), pp. 107-117.

[11] J. G. Wardrop, "Some Theoretical Aspects of Road Traffic Research", Proceeding of the Institute of Civil Engineers(Part II), (1952), pp. 325-378.

[12] Federal Highway Administration (FHWA), "Urban transportation planning system (UTPS)", US Department of Transportation, Washington,(1979).

[13] L. J. LeBlanc, E. K. Morlok and W. P. Pierskalla, "An Efficient Approach to Solving the Road Network Equilibrium Traffic assignment problem", Transportation Research, vol. 9, no. 5, (1975), pp. 309-318.

[14] M .Beckmann, C. B. McGuire and C. B. Winsten, "Studies in the Economics of Transportation", New Heaven (CT): Yale University Press, (1956).

[15] Y. Sheffi, "Urban transportation network", Pretince Hall, (1985).

[16] B. F. Si and Z. Y. Gao, Editor, "Flow Analysis of Traffic Transportation Network and Optimization Modeling", China Communications Press, Beijing, (2013)

[17] M. L. Hetland, "Proof Methods and Greedy Algorithms", Lecture notes, (2008).

[18] Y. W. Chen and G. H. Tzeng, “ A fuzzy multi-objective model for reconstructing the post-quake roadnetwork by genetic algorithm”, International Journal of Fuzzy Systems, vol. 1,no. 2, (1999), pp. 85-95.

\section{Authors}
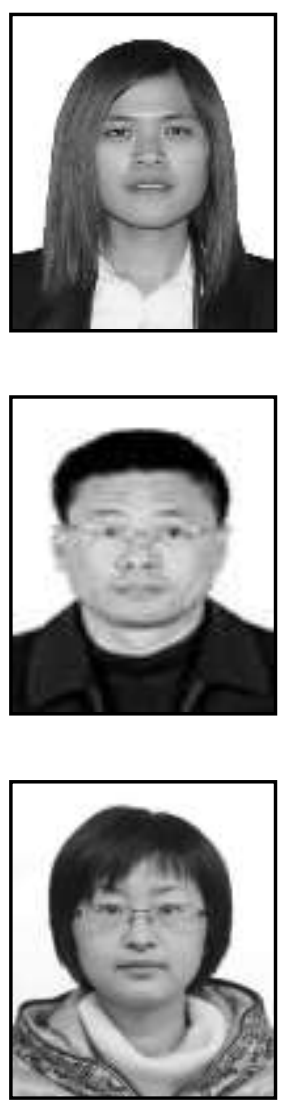

Yumei Li, she is a graduate. The main research field includes emergency logistics route optimization and traffic control scheme optimization.

Hongguo Wang he is a $\mathrm{PhD}$, Professor. The main research field includes e-government and logistics optimization.
Ping $\mathbf{L i}$, she is a PhD, Lecture. The main research field is network optimization. 


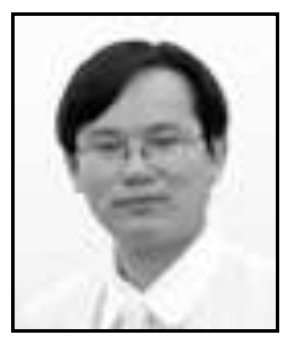

Zengzhen Shao, he is a $\mathrm{PhD}$, associate professor,the main research field is intelligent computing and route optimization.

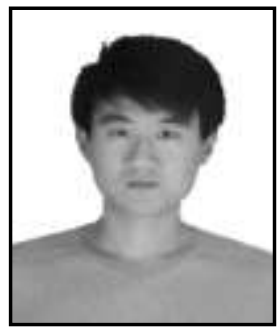

Mingguang Ma, he is a graduate. The main research field includes emergency logistics route optimization and traffic control scheme optimization. 